\title{
Vibrant Energy Aware Spray and Wait Routing in Delay Tolerant Network
}

\author{
Viren G. Patel, Tushar K. Oza, Dhavalsinh M. Gohil \\ Department of Computer science and Engineering, \\ S.P.B. Patel Engineering College, Saffrony, Mehsana, India \\ vickypatel87@gmail.com,tusharoza23@gmail.com \\ dhavalgohil@yahoo.com
}

\begin{abstract}
Delay tolerant networks (DTN) are wireless networks where disconnections arise often due to the mobility of nodes, failures of energy, the low density of nodes, or when the network extends over long distances. In these situations, traditional routing protocols that have been developed for mobile ad hoc networks prove to be unsuccessful to the scope of transmitting messages between nodes. The Spray and Wait routing may achieve low routing and energy efficiency due to the blindness in the spray phase. To deal with this situation, we propose an opportunistic routing with enclosed message copies, called the Vibrant Energy aware Spray and Wait (VESW), which utilizes the information about vibrancy of node and remaining energy to allocate the number of copies between the corresponding pair nodes in the spray phase.
\end{abstract}

Keywords: DTN, routing, vibrant spray and wait, energy aware, vibrancy of node

\section{Introduction}

Delay tolerant networks [1-3] are sparse multi-hop ad hoc networks in which nodes utilize any pair-wise contact opportunities to share and forward messages. There is not any preexisting internet infrastructure and most of the time it does not exist a complete route from the source to the destination in Delay tolerant networks. Therefore, Delay tolerant networks have to tolerate partitions, long disconnections and topology unsteadiness in general. The main challenge for this environment is that conventional routing schemes did not work properly.

At present the Internet architecture is based on the TCP / IP protocol. This structure carries out message forwarding in the IP network layer by means of IP protocol, and in the transmission layer it requires a certain pathway for a period of time. Traditional routing protocols will no longer be suitable to this new type of network architecture. On the other hand, Researchers have proposed a class of store-carry forward routing (Fig. 1) schemes $[4,5]$ for such network environment, where a node receives packets, stores them in their buffers, carries them while moving, and forwards them to other nodes when they encounter each other.

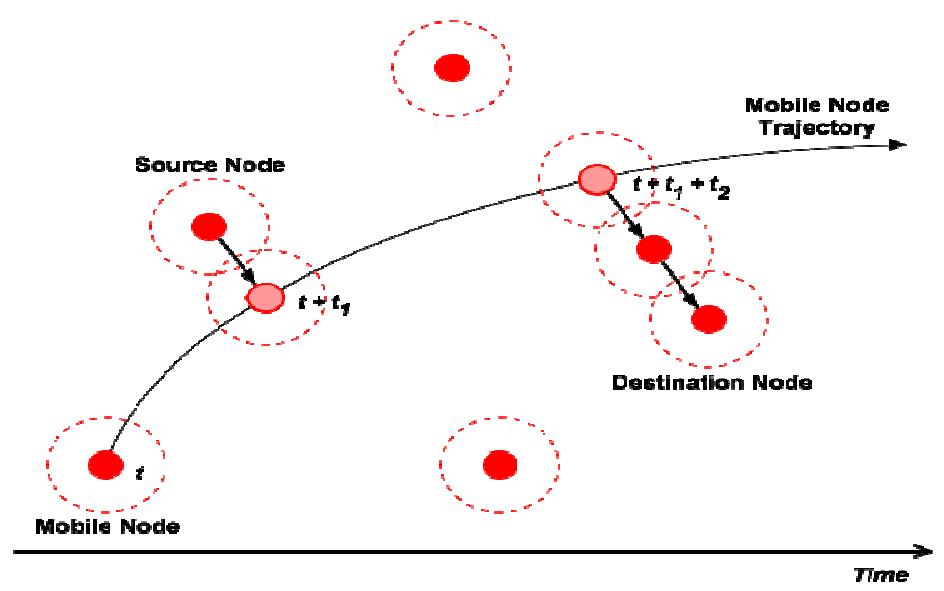

Figure 1. Store-carry forward routing 
This allows delivery among disconnected network components. Because nodes in this network are sparse, and the node transmission range is limited, nodes will need to deliver messages before messages are stored for a long time, moving through nodes and then forward messages to other relay nodes or the destination node.

The rest of the paper is structured as follows.

Section II goes over related works, describes several typical DTN routing protocols. Section III details the Vibrant Energy aware Spray and Wait (VESW). Section IV makes concludes the paper.

\section{Related works}

Since routing is one of the most compelling issues in opportunistic networks, several research groups are working on this topic. For the sake of space in this section we only mention Epidemic Routing [6], PROPHET [7], and Spray and WAIT (SW) [8], which are representative of fundamental approaches to routing in opportunistic network in [9].

\subsection{Epidemic Routing}

Epidemic Routing, nodes act as a relay for other nodes. Each node maintains a summary vector that indicates the set of packets which it has. When two nodes encounter within their transmission range, they exchange their summary vectors to check whether any packets are available, and basing on this information, each node can determine if other nodes have some packets that previously reach this node, and then they exchange packets which they don't have in common. Thus packets are disseminated in broadcast mode, as if infectious diseases spread, and at least packets are expected to reach the destination eventually. This packetspread will continue until all the nodes have a copy of the packet or its TTL (time-to-live) expires.

Although, Epidemic Routing achieves highest delivery of messages, but it makes no attempt to eliminate duplication, and the high delivery rate is at the expense of the network resource consumption, such as store buffer space and transmission bandwidth. VESW aims at drastically reduce the cost of such flooding by vibrant spray, Epidemic Routing uses to deliver a message is optimal, in the sense that it is the quickest one to deliver the message. EASW exploits vibrancy of node and particular route.

\subsection{Prophet}

Probabilistic Routing Protocol using History of Encounters and Transitivity (PROPHET) is an evolution of Epidemic Routing that introduced the concept of delivery predictability. Delivery predictability is the probability for a node to encounter a certain destination. PROPHET forwarding algorithm is similar to the Epidemic Routing one except that, during a contact, messages are requested only if the receiving node has greater delivery predictability for the destination. PROPHET is representative for a class of routing protocols that exploit some context information to limit the Epidemic Routing flood. EASW is able to manage and exploit far richer context information with respect to PROPHET.

\subsection{Spray and Wait (SW)}

Spray and Wait routing protocol, which includes the Source Spray and Wait and the Binary Spray and Wait. In Source Spray and Wait routing protocol, the simplest way is to have the source node forward all $L$ copies to the first $L$ distinct nodes it encounters. If all the first $L$ distinct nodes do not include the destination node, they will not transmit the message to the next hop until they meet the destination of this message. In the Binary Spray and Wait routing protocol, the source of a packet initially starts with $L$ copies; when a node $A$ (source node or relay node) that has $n>1$ message copies encounters a node $B$ (with no copies), it hands over to $B$ [n/2] and keeps [n/2] for itself; when node A has only one copy left, it switches to direct transmission and forwards the message only to its destination. When all nodes move in an IID (Independent and Identically Distributed) manner, the Binary Spray and Wait routing protocol has higher message delivery ratio and lower delay compared with the Source Spray and Wait routing protocol. But in real world environment, node mobility patterns are not in an IID manner. Although node mobility patterns are not entirely random, different node mobility patterns may follow their own laws of movement. Because the Binary Spray and Wait routing protocol gives 
half the message copies to the node it meets, it does not take into account differences in node activities. This algorithm forwards message copies with blindness, leading to low message delivery ratio and large overhead.

In the Spray and Wait, the copies of messages are sprayed with no consideration about any information like vibrancy of node, remaining energy, etc., that may lead to blind spray, energy aware, and low efficiency in data gathering.

\section{Vibrant Energy aware Spray and Wait (VESW)}

In Spray and Wait scheme Binary Spray and Wait (BSW) is proposed to speed up the spray phase and improve the routing performance. In the spray phase of BSW, any node A that has more than one message copy hands over to another node B half of its copies when it encounters node B. It's proved that BSW can minimize the spray time and achieve optimal performance under the condition that all nodes move in an IID manner. It will therefore serve as our baseline. However, remaining energy and vibrancy information of all nodes are not considered during the spraying of message copies that may result in blind spraying and low efficiency in data gathering.

For example, a node with higher Vibrancy of Node may encounter more nodes than the one with lower Vibrancy of Node during the same time interval. So the spray phase can be sped up if higher Vibrancy of Nodes carries more message copies. On the other hand, it may lead to higher risk of data collection if the node with less remaining energy is allocated with more message copies. Based on the above observations, a utility function Vibrancy of Node and remaining energy information is given here to speed up messages spreading in the spray phase and improve the routing efficiency.

\subsection{Delivery Utility}

When delivery rate stay the same, we consider the relationship between network overhead and delivery utility. Delivery utility is defined as the ratio of the number of messages received by destination nodes to the number of messages forwarded by relay nodes, as shown in Equation (1).

$$
\text { Delivery_Utility }=\frac{\text { Number of message received }}{\text { Number of message forwarded }}
$$

\subsection{The definition of Vibrancy of Node}

The Spray and Wait and The Epidemic routing protocols forward messages without taking node mobile patterns into thought, therefore the delivery ratio is too low. Now, we present the Vibrancy of Node. Vibrancy of Node indicates the activity of a node, or the number one node meets other different nodes within a given interval and remaining energy. In the same interval of time, the more nodes that one node meets, the greater the Vibrancy of Node. The Vibrancy of Nodes can dynamically represent the node activity in a given period of time. In this paper, we use the ratio of Vibrancy of Nodes to dynamically forward the number of message copies.

\subsection{Energy Consumption}

Energy consumption for each node transmits and receives data with fixed transmission and reception power, respectively. So the power consumption is independent of the transmission distance between adjacent nodes. Accordingly, we adopt the following energy model in [11-12] to calculate the power consumption.

$$
\mathrm{p} \approx \mathrm{e} .(\mathrm{Kr}+\mathrm{Kt})
$$

Where $p$ denotes the total energy consumption of one node for receiving $\mathrm{kr}$ bits and transmitting kt bits, and e is a factor indicating the energy consumption per bit at the receiver circuit.

JTI Vol. 1, No. 1, March 2013 : $43-47$ 


\subsection{Computation and update Vibrancy of Node}

Moving in the network, the node will encounter more and more nodes. In the real network situation we will add a number of new nodes from time to time. Some nodes have been in the network for a long time, whose Vibrancy of Node are larger. However, new nodes Vibrancy are smaller. Therefore, we divide time into a series of fragments at the same length. We calculate Vibrancy of Node on each fragment and consider the influence of the Vibrancy of Node in former fragment on the Vibrancy of Node in the current fragment. We divide time fragments into $n$.

$$
\sum_{i=1}^{n} \mathrm{ti}=\mathrm{T}, \quad \mathrm{t} 1=\mathrm{t} 2=\mathrm{t} 3 \ldots \ldots . . .=\mathrm{tn}
$$

With reference to Jacobson formula [10], the $\tilde{V}$ (Vibrancy of each Node) can be updated by Equation (4).

$$
\tilde{V}=\alpha \cdot \tilde{V} \text { old }+(1-\alpha) \cdot \text { Avg } \tilde{V}
$$

$\alpha$ is a smoothing factor, which determines the influence of the Vibrancy of Node in former fragment on the Vibrancy of Node in the current fragment. Vold refers to the Vibrancy of Node at the end time of former fragment and has to be updated regularly. In our algorithm we update the Vibrancy of Node once per Hour.

$$
\operatorname{Avg} \tilde{V}=\frac{K 2-K 1}{\Delta t} \cdot T
$$

In Equation (5), Avg $\tilde{V}$ is the number of other nodes that one node experiences in the network during the period of time from the end time of former fragment to the current time. Nodes must first update Avg $\tilde{V}$ and $\tilde{V}$ at each connection.

\subsection{Spray Strategy}

When two nodes encounter, they will update the Vibrancy of Nodes at first and exchange Vibrancy of Nodes with each other, and check the number of message copies $L$ on node $A$ is more than 1 , than algorithm will be run on node $A$ to calculate the number of copies sent to node $B$. And the spray phase continues until there is only one message copy left on node A.

After that, node A starts the wait phase to wait for direct communication opportunity with the final destination. The spray phase considers Vibrancy of Node and remaining energy information for disseminating message copies to delay the network lifetime, as a result, the opportunistic routing scheme proposed in the paper will be called Vibrant Energy-aware Spray and Wait (VESW).

As shown in (e.g. Figure 2), assuming that a node $A$ (source or relay) has $\mathrm{N} 1>1$ packet copies. When node $A$ encounters node $B$, the Vibrancy of node $A$ is $\tilde{V} 1$, remaining energy of node $A$ is $E 1$, the Vibrancy of node of node $B$ is $\tilde{V} 2$ and remaining energy of node $A$ is E2.

$$
\mathrm{N} 2=\frac{\tilde{\mathrm{V}} 2}{(\tilde{\mathrm{V}} 1+\tilde{\mathrm{V}} 2)} \cdot \frac{\mathrm{E} 2}{(\mathrm{E} 1+\mathrm{E} 2)} \cdot \frac{\mathrm{N} 1}{2}
$$

\subsection{Wait Strategy}

According the assumption about the Random Waypoint mobility, all nodes move independently. It will not do great help for improvement of delivery probability but lead to extra delivery overhead and energy consumption using the encounter history information in opportunistic routing. When a node has only one copy left, it switches to direct transmission, means that it will forward this message only to its destination. 


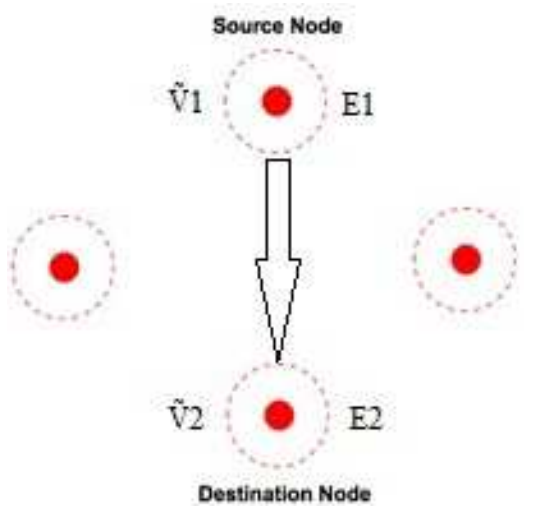

Figure 2. Forward Strategy

\section{Conclusion}

In this paper, we propose a vibrant energy-aware spray and wait routing scheme called VESW to improve energy efficiency of Spray and Wait routing. In VESW, an effectiveness function vibrancy of node and remaining energy information are presented to assign the number of copies between each related pair and avoid the blindness in the spray strategy of Spray and Wait. The proposed VESW routing outperforms BSW routing protocol in terms of energy aware, reduce network overhead, increase the delivery rate and improves the delivery utility.

\section{References}

[1] Pelusi L, Passarella A, Conti M. Opportunistic networking: data forwarding in disconnected mobile ad hoc networks. Communications Magazine. 2006; 44(11): 134-14.

[2] K Fall. A delay-tolerant network architecture for challenged internet. In Proc. ACM SIGCOMM. 2003:24-27.

[3] Akyildiz IF, Akan B, Chen C, Fang J, and Su W. Inter- Planet Network Internet: State-of-the-Art and research challenges. Computer Networks. 43(2):75-112.

[4] Pei Chun Cheng, Jui Ting Weng, Lung Chih Tung, Kevin Lee, Mario Gerla, Jerome Haerri, "GeoDTN+Nav: A Hybrid Geographic and DTN Routing with Navigation Assistance in Urban Vehicular Network". MobiQuitous/ISVCS 2008, Trinity College Dublin, Ireland. 2008.

[5] Wu and S Yang. "Logarithmic Store-Carry-Forward Routing in Mobile Ad Hoc Networks". IEEE Transactions on Parallel and Distributed Systems. 2007.

[6] A Vahdat and D Becker. "Epidemic routing for partially connected ad hoc networks". Tech. Rep. CS2000-06, CS Dept., Duke University. 2000.

[7] A Lindgren, A Doria, and 0 Schelen. "Probabilistic routing in intermittently connected networks". ACM Mobile Computing and Communications Review. 2003; 7(3).

[8] M Musolesi, S Hailes, and C Mascolo. "Adaptive Routing for Intermittently Connected Mobile Ad Hoc Networks". Proc. Of IEEE WoWMoM. 2005.

[9] L Pelusi, A Passarella, and M Conti. "Opportunistic Networking: data forwarding in disconnected mobile ad hoc networks". IEEE Communications Magazine. 2006; 44(11).

[10] Prentice Hall Andrew S Tanenbaum. Computer Networks, Foutth Edition. Peking, Press of Tsinghua University. 2007, P551.

[11] $M$ Wang, $S$ Basagni, et al. Exploiting sink mobility for maximizing sensor networks lifetime. Proc. of the 38th Annual Hawaii Int'l Conf. on System Sciences. Hawaii: IEEE Computer Society. 2005; 287a287a.

[12] S Gao, $H$ Zhang, et al. Efficient data collection in wireless sensor networks with path-constrained mobile sinks. Proc. of the 10th IEEE Int'l Symp. on a World of Wireless, Mobile and Multimedia Networks (WoWMoM). 2009

[13] J Kevin Fall. Applications, "A delay-tolerant network architecture for challenged internets". Proceedings of the 2003 conference on Applications, technologies, architectures, and protocols for computer communications SESSION. Position papers Pages: $27-34$.

[14] TKK/COMNET, sProject page of the ONE simulator, http//www.netlab.tkk.fi/tutkimus/dtn/theone/, 2008.

JTI Vol. 1, No. 1, March 2013: $43-47$ 